\title{
Simultaneous Double Star and Cluster FTEs observations on the dawnside flank of the magnetosphere
}

\author{
A. Marchaudon ${ }^{1}$, C. J. Owen ${ }^{1}$, J.-M. Bosqued ${ }^{2}$, R. C. Fear ${ }^{1}$, A. N. Fazakerley ${ }^{1}$, M. W. Dunlop ${ }^{3}$, A. D. Lahiff ${ }^{1}$, \\ C. Carr $^{4}$, A. Balogh ${ }^{4}$, P.-A. Lindqvist ${ }^{5}$, and H. Rème ${ }^{2}$ \\ ${ }^{1}$ Mullard Space Science Laboratory, University College London, Holmbury St Mary, Dorking, RH5 6NT, UK \\ ${ }^{2}$ Centre d'Etude Spatiale des Rayonnements, CESR/CNRS, B.P. 4346, 31028 Toulouse Cedex, France \\ ${ }^{3}$ Rutherford-Appleton Laboratory, Chilton, Didcot, Oxon, OX11 0QX, UK \\ ${ }^{4}$ Space and Atmospheric Physics, Blackett Laboratory, Imperial College, London, SW7 2BZ, UK \\ ${ }^{5}$ Alfvén Laboratory, Royal Institute of Technology, Stockholm, 10044, Sweden
}

Received: 23 February 2005 - Revised: 6 May 2005 - Accepted: 26 May 2005 - Published: 8 November 2005

Part of Special Issue "Double Star - First Results"

\begin{abstract}
We present Cluster and Double Star-1 (TC-1) observations from a close magnetic conjunction on 8 May 2004. The five spacecraft were on the dawnside flank of the magnetosphere, with TC-1 located near the equatorial plane and Cluster at higher geographic latitudes in the Southern Hemisphere. TC-1, at its apogee, skimmed the magnetopause for almost 8 h (between 08:00-16:00 UT). Flux Transfer Events (FTEs), moving southward/tailward from the reconnection site, were observed by TC- 1 throughout almost all of the period. Cluster, travelling on a mainly dawn-dusk trajectory, crossed the magnetopause at around 10:30 UT in the same Magnetic Local Time (MLT) sector as TC-1 and remained close to the magnetopause boundary layer in the Southern Hemisphere. The four Cluster spacecraft observed FTEs for a period of $6.5 \mathrm{~h}$ between 07:30 and 14:00 UT.
\end{abstract}

The very clear signatures and the finite transverse sizes of the FTEs observed by TC- 1 and Cluster imply that, during this event, sporadic reconnection occurred. From the properties of these FTEs, the reconnection site was located northward of both TC-1 and Cluster on the dawn flank of the magnetosphere. Reconnection occurred between draped magnetosheath and closed magnetospheric field lines. Despite variable interplanetary magnetic field (IMF) conditions and IMF- $B_{z}$ turnings, the IMF clock angle remained greater than $70^{\circ}$ and the location site appeared to remain relatively stable in position during the whole period. This result is in agreement with previous studies which reported that the dayside reconnection remained active for an IMF clock angle greater than $70^{\circ}$. The simultaneous observation of FTEs at both Cluster and TC-1, separated by $2 \mathrm{~h}$ in MLT, implies that the reconnection site on the magnetopause must have been extended over several hours in MLT.

Correspondence to: A. Marchaudon

(am@mssl.ucl.ac.uk)
Keywords. Magnetospheric physics (Magnetospheric configuration and dynamics; Solar wind-magnetosphere interaction) - Space plasma physics (Magnetic reconnection)

\section{Introduction}

Magnetic reconnection between magnetospheric and interplanetary magnetic fields (IMF) is a commonly accepted process, allowing transfer of energy and momentum from the solar wind to the magnetosphere. Many models and observations have shown different possible topologies for this process. Reconnection can occur between strictly anti-parallel field lines, at the nose of the magnetopause during dominant southward IMF (Dungey, 1961; Crooker, 1979; Lockwood et al., 1990). In addition, as a consequence of the IMF draping, magnetopause reconnection may also occur on the lobes during dominant northward IMF (Dungey, 1963; Maezawa, 1976; Gosling et al., 1991) or on the flanks during periods of dominant azimuthal IMF component (Phan et al., 2000, 2001; Marcucci et al., 2000). Component reconnection between magnetic fields with a relatively low shear can also occur almost everywhere on the dayside magnetopause (Sonnerup, 1974; Chandler et al., 1999). Indeed, observations of component reconnection equatorward of the cusp have been reported during periods of northward IMF (Fuselier et al., 2000). Moreover, the coexistence of high-latitude (lobe) and low-latitude reconnection sites has been observed (Sandholt et al., 1998; Pitout et al., 2002). Finally, simulations by Berchem et al. (1995) and recent observations by Vaisberg et al. (2004) suggested the possible existence of multiple Xlines forming isolated magnetic flux ropes on the dayside, as well as on the flanks of the magnetopause, for various IMF conditions. These results support the multiple reconnection lines model of Lee and $\mathrm{Fu}(1985)$. 
The reconnection process is very complex. In particular, the large-scale spatial and temporal nature of the reconnection is still poorly understood. Various observations from satellites and/or ground-based stations have shown that a reconnection line can be stable and extended, by up to $40 R_{E}$, along the magnetopause (Milan et al., 2000; Phan et al., 2000, 2001; Pinnock et al., 2003), especially during stable IMF conditions. However, how the reconnection line evolves during variable IMF conditions remains an important question. A large number of studies have shown that reconnection is essentially a sporadic phenomenon, forming small-scale flux tubes called flux transfer events (Russell and Elphic, 1978; Pinnock et al., 1995; Bosqued et al., 2001; Owen et al., 2001). The question of whether reconnection has an intrinsic sporadic nature, or whether it is controlled by internal magnetospheric conditions (Russell et al., 1997) or by variable IMF conditions (Lockwood and Wild, 1993), is still open. Some authors have shown that during very steady IMF conditions, reconnection can occur continuously, but at a variable rate (Gosling et al., 1982; Phan et al., 2000, 2001, 2004).

We thus need to develop an understanding of how variations of the IMF and/or magnetospheric conditions can change the topology and the properties of magnetopause reconnection. Measurements from several satellites located on different parts of the magnetopause, together with associated ground-based observations and simulations, will be needed to answer this question. As an initial example of this type of study, Wild et al. (2005) investigated simultaneous observations of FTEs by both Geotail and Cluster, located, respectively, at low- and high-latitudes on the magnetopause, and inferred the magnetopause reconnection geometry. In this paper, we study an excellent conjunction between Cluster and Double Star-1 on 8 May 2004. The five spacecraft were all located in the Southern Hemisphere and simultaneously observed FTEs over a period of several hours. The different parts of the magnetopause probed by Cluster (high-latitude) and Double Star-1 (near-equatorial latitude) and the use of Cluster multi-spacecraft analysis allow us to develop an understanding of the reconnection geometry on the dawn flank magnetopause during this period and to document the effect of variable IMF conditions.

\section{Instrumentation}

Cluster has an elliptical polar orbit with a perigee of $\sim 4 R_{E}$, an apogee of $\sim 19 R_{E}$ and a period of $\sim 58 \mathrm{~h}$. Double Star1 (TC-1) has an elliptical equatorial orbit with a perigee of $\sim 0.1 R_{E}$, an apogee of $\sim 12 R_{E}$ and a period of $\sim 14 \mathrm{~h}$. In this study, data from several plasma and field experiments on the Cluster and Double Star satellites are used. The Plasma Electron and Current Experiment (PEACE) (Johnstone et al., 1997; Fazakerley et al., 2005) provides the electron velocity distribution every $4 \mathrm{~s}$ (spacecraft spin period), in the energy range from $0.7 \mathrm{eV}$ to $\sim 30 \mathrm{keV}$. Moments of the full threedimensional distribution are obtained with a resolution up to 4 s. The Hot Ion Analyser (HIA) (Rème et al., 2001), which offers a good energy and angular resolution without mass resolution, provides a full three-dimensional energy/velocity distribution of ions (protons) from thermal energies up to about $32 \mathrm{keV} / \mathrm{q}$, and moments also with a time resolution up to $4 \mathrm{~s}$. The Flux Gate Magnetometer (FGM) (Balogh et al., 2001) measures the 3-D magnetic field vector. In this paper, we use data at 4-s resolution. All these instruments are similar on Cluster and Double Star, except for the PEACE experiment, for which two sensors are installed on Cluster but only one on Double Star (see Fazakerley et al., 2005). In this study, we present energy spectrograms and moment data (density, temperature and velocity components of the ions and electrons) provided by the PEACE and the HIA experiments on board Double Star and Cluster.

Finally, solar wind plasma and magnetic field data were obtained from the Solar Wind Experiment (SWE) and the Magnetic Fields Investigation (MFI) of the Wind satellite.

\section{Geometry of the conjunction and interplanetary con- ditions}

The trajectories of Double Star/TC-1 and Cluster/sc1 are presented in Fig. 1, in the $X Y$ and $Y Z$ GSM planes. The position of a modelled magnetopause (Shue et al., 1997), plotted, respectively, in the $Z_{G S M}=0$ and $X_{G S M}=0$ planes, is also superimposed. This empiric model is parameterised by the solar wind dynamic pressure and the $B_{z}$ component of the IMF. We have chosen averaged values on the 08:00-16:00 UT period of the solar wind pressure and of the IMF- $B_{z}$ component, deduced from the Wind satellite data $\left(P_{S W}=1.4 \mathrm{nPa}\right.$ and $\left.B_{z}=-1.6 \mathrm{nT}\right)$. Between $08: 00$ and 16:00 UT, TC-1 was located just southward of the equatorial plane $\left(Z_{G S M} \sim-3 R_{E}\right)$ and skimmed the dawnside magnetopause around $Y_{G S M} \sim-12 R_{E}$, travelling about $4 R_{E}$, predominantly in the $+X_{G S M}$ direction. On the other hand, the four Cluster spacecraft entered the magnetosphere around 10:30 UT, at high geographic latitudes in the Southern Hemisphere and slightly downstream of the TC-1 position. Over the next few hours, the four spacecraft penetrated deeper into the magnetosphere, moving predominantly in the $-Y_{G S M}$ direction, at around $X_{G S M} \sim-1 R_{E}$ and $Z_{G S M} \sim-10 R_{E}$.

The interplanetary conditions were monitored by the Wind satellite, situated upstream, southward and dawnward of the magnetosphere $\left(X_{G S M}=96 R_{E}, Y_{G S M}=-25 R_{E}\right.$, and $Z_{G S M}=-19 R_{E}$ ). For this study, the magnetic data have been lagged by $23 \mathrm{~min}$, to take into account the propagation of the solar wind from Wind's position to the Earth's magnetosphere (with a solar wind bulk speed of $\sim 480 \mathrm{~km} \mathrm{~s}^{-1}$ ). The IMF components in GSM, as well as the clock angle $\left(\arctan \left[\left|B_{y}\right| / B_{z}\right]\right)$, are plotted in Fig. 2, between 07:00 and 16:00 UT. The interplanetary magnetic field orientation was variable until 11:00 UT and then became more stable. The IMF- $B_{x}$ component (panel 2a) was strongly positive at the beginning of the period, but decreased from +6 to $0 \mathrm{nT}$ until 11:00 UT and then varied between $\pm 2 \mathrm{nT}$. Before 09:00 UT and after 11:00 UT, IMF- $B_{y}$ (panel 2b) was negative and very 


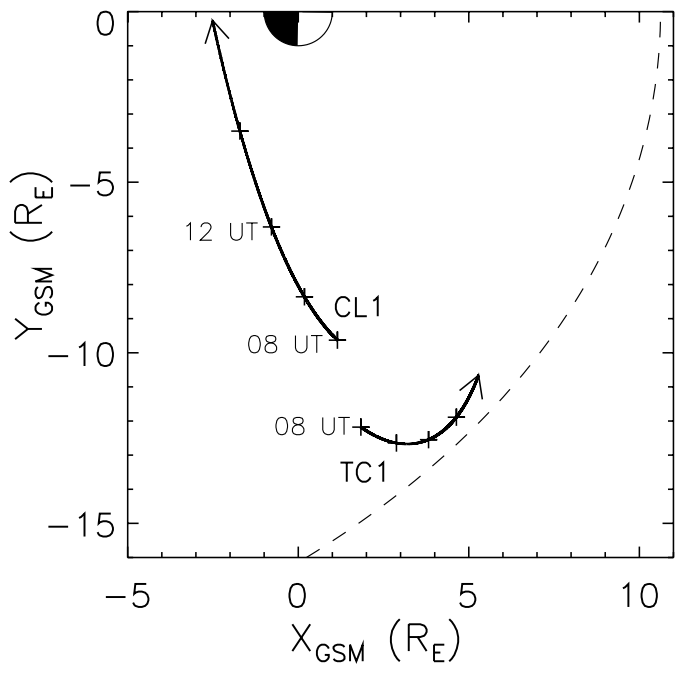

(a)

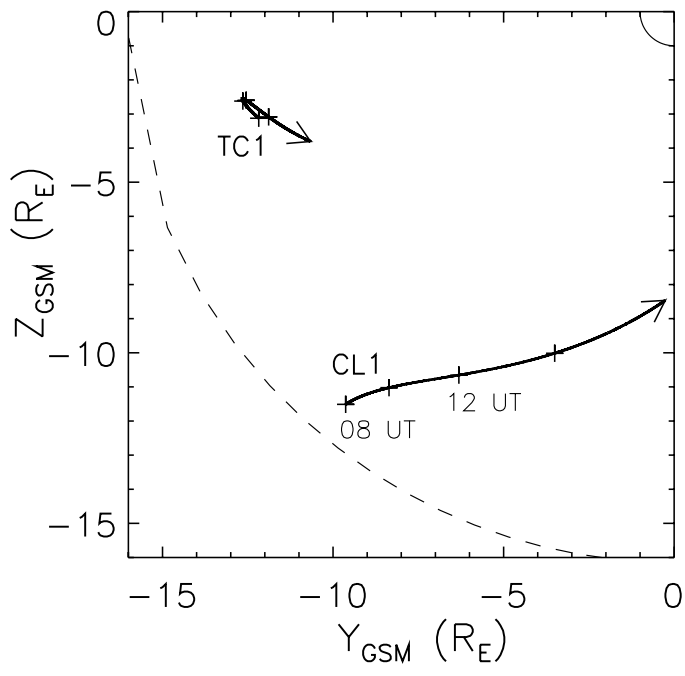

(b)

Fig. 1. Trajectories of Cluster/sc1 and TC-1 for the period 08:00-16:00 UT, in GSM coordinates. (a) in the $X Y$ plane, (b) in the $Y Z$ plane. Black crosses along the orbits are separated by $2 \mathrm{~h}$. A model of the magnetopause position (see text for details) is indicated in the $Z_{G S M}=0$ plane, panel (a), and in the $X_{G S M}=0$ plane, panel (b), by the dashed line.

stable at around $-5 \mathrm{nT}$. In between these times, $B_{y}$ was more variable between -2 and $0 \mathrm{nT}$, with a strong positive excursion at 10:20-10:30 UT. Before 08:20 UT, IMF- $B_{z}$ (panel 2c) was slightly positive around $+1 \mathrm{nT}$, turned negative $(\sim-4 \mathrm{nT})$ between 08:20 UT and 11:00 UT and then varied between +1 and $-4 \mathrm{nT}$ until the end of the period. Despite all these variations, the IMF clock angle (panel 2d) remained greater than, or approximately equal to, $70^{\circ}$, except some very short excursions at around 07:30 and 08:15 UT. On the other hand, the plasma parameters of the solar wind were very stable during the entire period, with a steady solar wind dynamic pressure of $\sim 1.4 \mathrm{nPa}$ (not shown).

\section{Observations}

\subsection{TC-1 observations}

\section{a) Overview of the data}

For the period 08:00-16:00 UT, data from the TC-1 satellite are presented in Fig. 3, together with the Wind IMF clock angle, for ease of comparison (top panel a). Panels (b, c) and (d) show energy flux spectrograms provided by the PEACE instrument, for electrons moving in the directions parallel, perpendicular and anti-parallel to the local magnetic field, respectively. Panel (e) shows the omnidirectional energy flux ion spectrogram provided by the HIA instrument. Panels (f) and $(\mathrm{g})$ display the electron density and temperature with $8 \mathrm{~s}$ resolution. Finally, panels ( $\mathrm{h}-\mathrm{k}$ ) give the 3 components of the FGM magnetic field transformed in the $\mathrm{L}, \mathrm{M}, \mathrm{N}$ coordinate frame (Russell and Elphic, 1978) and its magnitude. In this frame, the $B_{N}$ component is normal to the magnetopause, the $B_{L}$ component lies in the magnetopause plane and is parallel

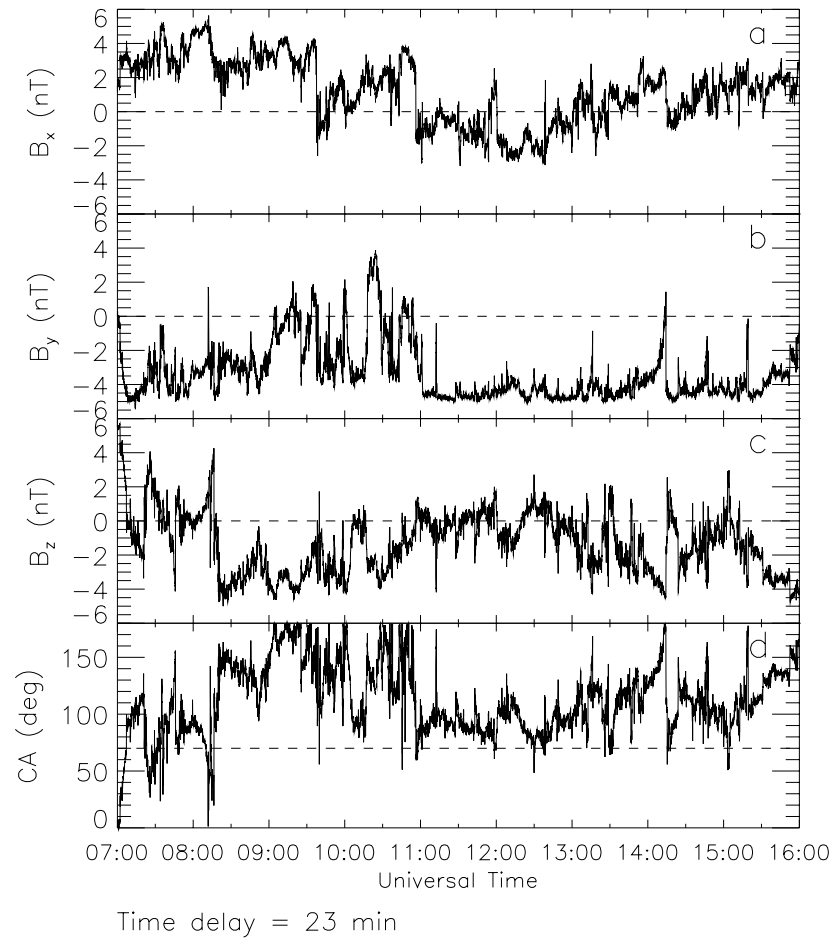

Fig. 2. Wind IMF data in GSM coordinates, lagged with $23 \mathrm{~min}$, for the period 07:00-16:00 UT. Panels (a), (b), (c) show the $B_{x}, B_{y}$ and $B_{z}$ components of the IMF and panel (d) shows the IMF clock angle.

to the projection of the $+Z_{G S M}$ axis on that plane. Finally, the $B_{M}$ component completes a right-hand set. In the case of TC-1, several magnetopause crossings were observed between 08:00 and 16:00 UT. The magnetopause normals at 


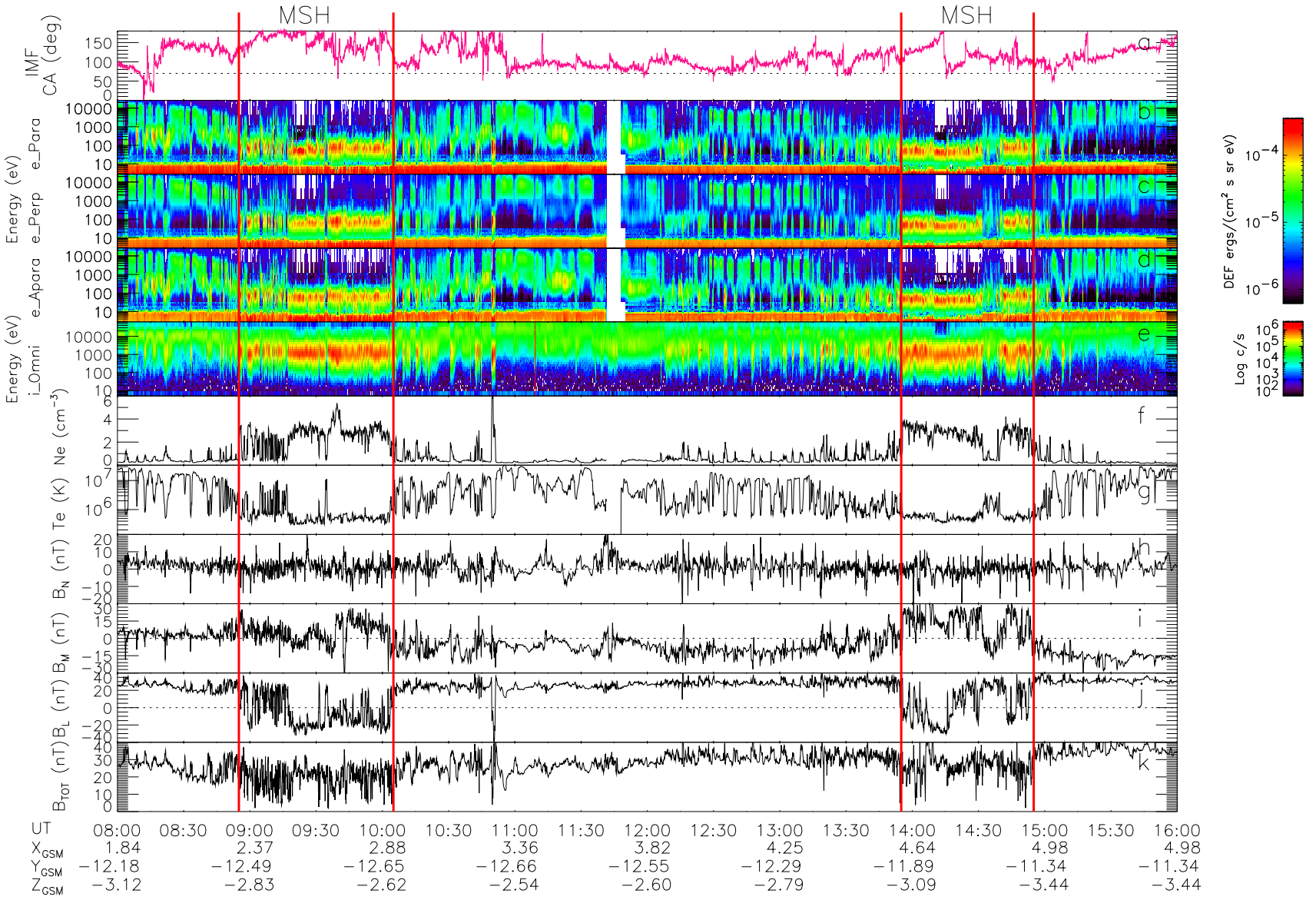

Fig. 3. Wind and Double Star TC-1 data for the period 08:00-16:00 UT. (a) IMF clock angle obtained from the Wind data and lagged with $23 \mathrm{~min}$, (b) to (d) PEACE electron spectrograms in the parallel, perpendicular and anti-parallel directions, (e) HIA omnidirectional ion spectrogram, (f) PEACE electron density, (g) PEACE electron temperature, (h) to (k) $B_{L}, B_{M}, B_{N}$ components and field magnitude of the FGM magnetic field.

each crossing were quite similar throughout the period and the ratios between intermediate and minimum eigenvalues were relatively high $(\sim 5)$. To select a representative $\mathrm{L}, \mathrm{M}$, $\mathrm{N}$ frame, we chose the normal from the 10:05 UT magnetopause crossing.

Throughout the period, TC-1 skimmed the dawnward magnetopause essentially on the magnetospheric side, but made two magnetosheath incursions between 08:50 and 10:05 UT and between 13:55 and 14:55 UT, characterised by dramatic changes in the plasma properties and very clear magnetic field rotations, typical of magnetopause crossings. Inside the magnetosphere, the plasma was hot and tenuous (panels $3 \mathrm{~b}-\mathrm{e}$ ). The magnetic field was essentially pointing northward $\left(B_{L}>0\right)$ and, as TC-1 progressed sunward on its orbit, the magnetic field direction became more sunward $\left(B_{M}<0\right)$ (panels $3 \mathrm{i}$ and $\mathrm{j}$ ). The magnetospheric plasma velocity was very low, but slightly sunward (not shown). In the magnetosheath, the plasma was cold and dense, and the magnetic field was directed southward $\left(B_{L}<0\right)$ and tailward $\left(B_{M}>0\right)$, consistent with a negative IMF- $B_{y}$ draped around the magnetosphere and a mainly negative IMF- $B_{z}$ during these periods. The magnetosheath velocity (not shown) was typically directed tailward, dawnward and southward $\left(\sim 250 \mathrm{~km} \mathrm{~s}^{-1}\right)$. During the overall period between 08:00 and 16:00 UT, TC-1 observed clear signatures of reconnection, described in the paragraph below, except between 10:55 and 12:05 UT, when TC-1 appeared to move slightly deeper into the magnetosphere and was therefore not well located to observe reconnection activity on the magnetopause.

\section{b) Detailed description of the reconnection signatures}

Typical reconnection signatures were observed by TC-1, both in the magnetosphere and in the magnetosheath sides. The signatures were clearer in the magnetosphere, but showed the same properties in the two regions. A majority of the observed structures were characterised by a mixing of magnetospheric and magnetosheath plasma (panels 3be), field-aligned streaming of electrons (panels $3 \mathrm{~b}$ and d), an increase (decrease) in the electron density and a decrease (increase) in the electron temperature (panels $3 \mathrm{f}$ and $\mathrm{g}$ ), with respect to magnetospheric (magnetosheath) values. The observed structures were also characterised by an increase in the $B_{L}$ magnetic field component (panel $3 \mathrm{j}$ ) and in the 
magnetic field magnitude (panel $3 \mathrm{k}$ ), by a decrease of the $B_{M}$ component (panel $3 \mathrm{i}$ ), and more significantly, by a "reverse" bipolar signature of the $B_{N}$ component (a negative, followed by a positive excursion) (panel $3 \mathrm{~h}$ ).

The four top panels of Fig. 4 show an expansion of the TC1 data, when the spacecraft was located in the magnetosphere (12:10-12:40 UT). Panel (a) shows the PEACE electron density, panels (b) and (c) show, respectively, the $B_{N}$ and the magnitude of the FGM magnetic field, and panel (d) shows the 3 components of the CIS-HIA ion velocity, in GSM coordinates. This expansion clearly highlights the features of the reconnection signatures: an increase in the electron density (panel 4a), "reverse" bipolar signature of the $B_{N}$ magnetic field component (panel $4 \mathrm{~b}$ ) and increase of the total magnetic field (panel 4c). In the magnetosphere, the velocity was low and slightly sunward. Inside the reconnected structures, the ion velocity turned to the same direction as the magnetosheath velocity (tailward, dawnward and southward), but to an amplitude higher than that in the contiguous magnetosheath (panel 4d). All these signatures are typical of FTE structures (Russell and Elphic, 1978; Paschmann et al., 1982; Berchem and Russell, 1984; Farrugia et al., 1988) and will be discussed in more detail in Sect. 5. The signatures of reconnection (as described above) remained similar throughout the $8 \mathrm{~h}$, in which TC-1 skimmed the magnetopause.

\subsection{Cluster data}

\section{a) Overview of the data}

The Cluster/spacecraft $4(\mathrm{sc} 4)$ data are presented in Fig. 5 for the period 07:30-14:00 UT. For convenience, panel (a) again shows the Wind IMF clock angle for this period. We chose sc4, because this is the only spacecraft for which the PEACE experiment was switched on before 09:30 UT and the full 3-D electron distribution was available with a 16-s resolution, enabling calculation of ground moments at this resolution. Panels (b-d) show electron spectrograms in the parallel, perpendicular and anti-parallel directions. Panel (e) gives the electron density in a logarithmic scale to highlight the density variations in the boundary layer. Panels $(\mathrm{f}-\mathrm{h})$ show the three components of the magnetic field in the $\mathrm{L}$, $\mathrm{M}, \mathrm{N}$ system. As no clear magnetopause crossing was observed in the magnetic data, in order to define the L, M, $\mathrm{N}$ frame, we chose a magnetopause normal deduced from the Roelof and Sibeck (1993) model at the Cluster position, where the magnetopause crossing was detected in the plasma data (10:30 UT). Finally, panel (i) shows the magnitude of the magnetic field for all four spacecraft (sc1 in black, sc2 in red, sc3 in green and sc4 in blue), which can be used to discriminate between the spatial and temporal variations. During the entire period 07:30-14:00 UT, the four Cluster spacecraft were in an approximately co-linear formation, with sc1, sc4 and sc2 leading and sc 3 trailing with a slight delay. The spacecraft were aligned essentially along the $Y_{G S M}$ direction, with a separation of $\sim 6000 \mathrm{~km}$ between the leading and the trailing spacecraft (sc1 and sc3, respectively).
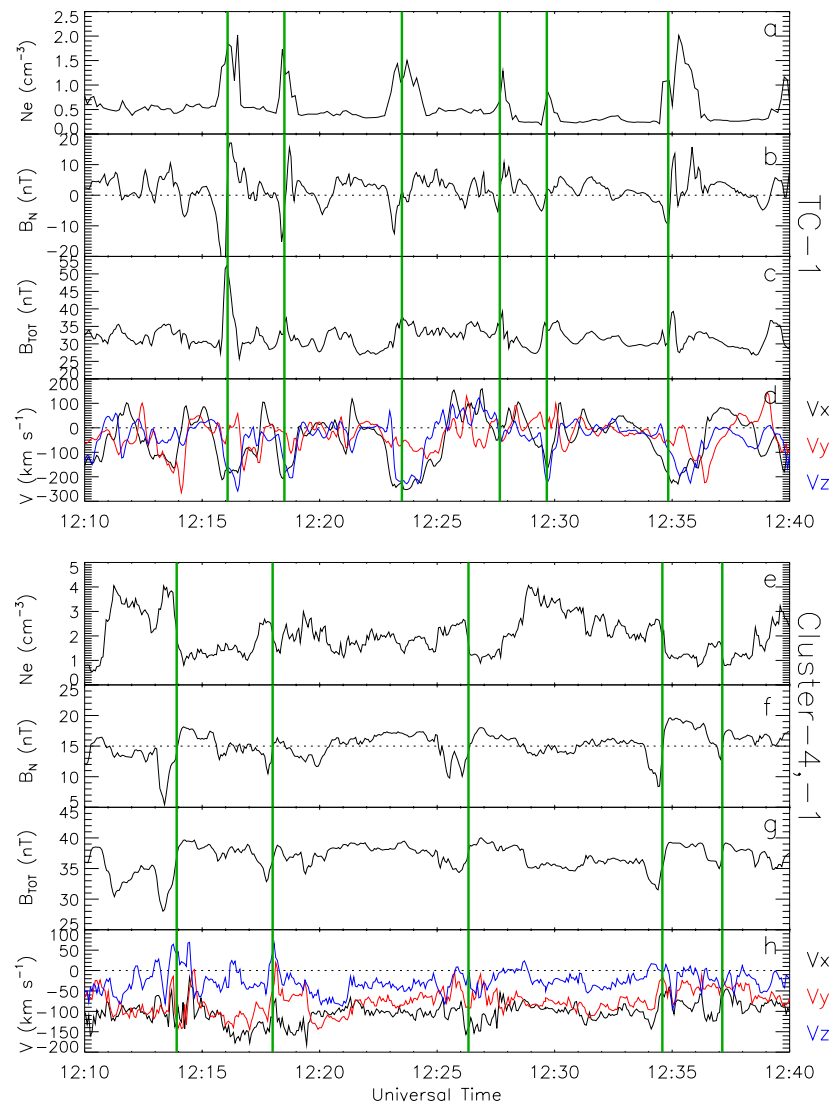

Fig. 4. Zoom in of the TC-1 (four top panels) and Cluster (sc1 and sc4) (four bottom panels) data, for the 12:10-12:40 UT. The data sets are identical for TC-1 and Cluster. Panel (a) shows the PEACE/TC-1 electron density, panels (b) and (c) show, respectively, the $B_{N}$ component and the magnitude of the FGM/TC-1 magnetic field and panel (d) shows the 3 components of the CISHIA/TC-1 ion velocity, in GSM coordinates. Panel (e) shows the PEACE/sc4 electron density, panels (f) and (g) show, respectively, the $B_{N}$ and the magnitude of the FGM/sc4 magnetic field and panel (h) shows the 3 components of the CIS-HIA/sc1 ion velocity, in GSM coordinates.

Before 10:30 UT at sc1 and sc4 and before 11:00 UT at $\mathrm{sc} 2$ and $\mathrm{sc} 3$, the variations of the $B_{y}$ and $B_{z}$ magnetic field components measured by the FGM experiment were nearly identical to the variations of the $B_{y}$ and $B_{z}$ components of the IMF measured by the Wind satellite. This suggests that the Cluster spacecraft were within the magnetosheath at these times, as supported by the magnetosheath-like plasma observed by PEACE (panels 5b-d). The Cluster spacecraft then successively entered the Southern Hemisphere dawnside magnetosphere, in the order 1-4-2-3. The magnetopause crossing for each spacecraft was very clearly identified by the plasma changes, at 10:30 UT for sc1 and sc4 (panels 5b-d) and at 11:00 UT for sc2 and sc3. Unfortunately, the magnetic rotation observed by each spacecraft during the magnetopause crossing was identical to the IMF- $B_{y}$ variation. A possible explanation for this lack of clear magnetic field rotation during the magnetopause 


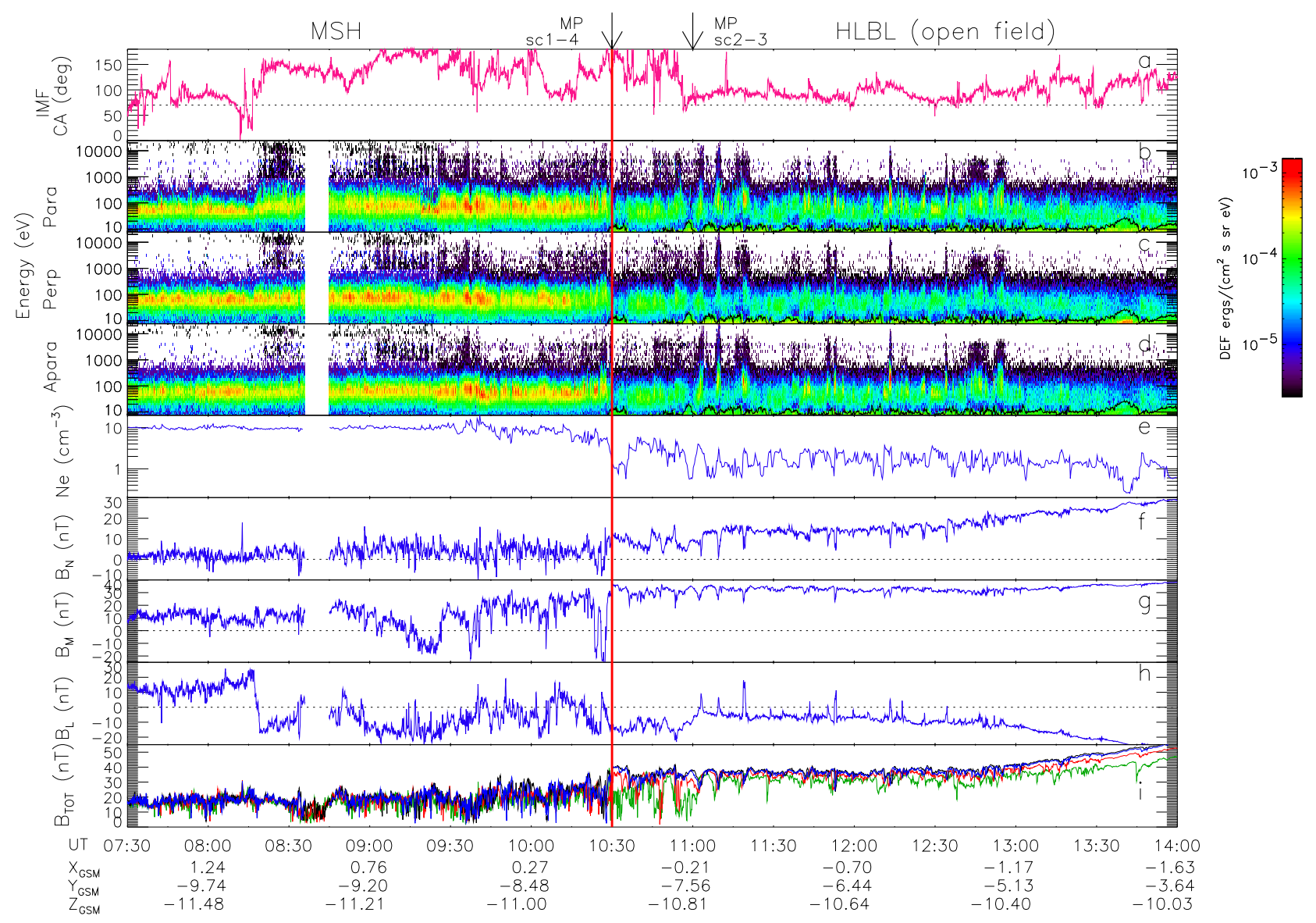

Fig. 5. Wind and Cluster data for the period 07:30-14:00 UT. (a) IMF clock angle obtained from the Wind data and lagged with $23 \mathrm{~min}$, (b) to (d) PEACE/sc4 electron spectrograms in the parallel, perpendicular and anti-parallel directions (flux below $2.4 \times 10^{-6} \mathrm{ergs} \mathrm{cm}^{-2} \mathrm{~s}^{-1} \mathrm{sr}^{-1} \mathrm{eV}^{-1}$ has been removed), with the EFW spacecraft potential superimposed (e) PEACE/sc4 electron density plotted with a logarithmic scale, (f) to (h) $B_{L}, B_{M}, B_{N}$ components of the FGM/sc4 magnetic field (i) FGM magnetic field magnitude for the four spacecraft (sc1-black, sc2-red, sc3-green, sc4-blue).

crossing itself could be explained by the relatively low shear between the magnetosheath and magnetospheric fields in this flank region and/or by the IMF variations hiding the magnetopause magnetic field rotation. After 10:30-11:00 UT, the four Cluster spacecraft skimmed the inner side of the magnetopause, remaining in the southern High-Latitude Boundary Layer (HLBL), characterised by a more tenuous plasma than in the magnetosheath (panels $5 \mathrm{~b}-\mathrm{d}$ ). At about 14:00 UT, they finally entered the lobe proper. Throughout the time interval, between 07:30 UT and 14:00 UT, the PEACE instruments on board Cluster detected numerous bursty signatures of field-aligned electron injections. After 09:20 UT, these plasma observations were clearly associated with bipolar signatures in the normal magnetic field $B_{N}$ component recorded by FGM.

\section{b) Detailed description of the injection signatures}

In the dawnside Southern Hemisphere magnetosheath (07:30-10:30 UT), PEACE/sc4 observed a cold, dense and quasi-isotropic plasma (panels 5.b-d). Again, signatures of reconnection were evidenced by the electron data and changed gradually when Cluster approached the magnetosphere. Between 07:30 and 08:00 UT, the electron injections were characterised by a highly anisotropic magnetosheathlike plasma, heated in the parallel direction (up to $1 \mathrm{keV}$ ), with respect to the surrounding plasma (panel 5b). During this first period, no clear magnetic signatures could be associated with these plasma injections. No signatures were observed between 08:00 and 08:20 UT. Between 08:20 and 10:30 UT, the detected plasma injections were a mixture of high-energy, omnidirectional plasma of magnetospheric origin (up to $10 \mathrm{keV}$ ) and low-energy plasma of magnetosheath origin (panels $5 \mathrm{~b}-\mathrm{d}$ ). However, clear associated magnetic signatures were not evident, except for some "reverse" bipolar signatures in the $B_{N}$ component. These $B_{N}$ signatures were not accompanied by an observable increase in the magnetic field magnitude.

In the magnetosphere (10:30-14:00 UT), electron injections were still observed, along with the more tenuous boundary layer plasma. These injections were characterized by a mixture of high-energy, omnidirectional plasma and, now, 
of low-energy plasma, mainly bi-directionally field-aligned, slightly heated with respect to the magnetosheath plasma (up to $\sim 1 \mathrm{keV}$ ), and by a slight increase in the electron density (panels 5b-e). After 10:30 UT, the Cluster spacecraft entered more deeply into the magnetosphere and an almost continuous increase in the magnetic field strength was clearly observed until 14:00 UT (panel 5i), even on the $B_{N}$ component (panel 5f). However, it is worth noticing that clear "reverse" bipolar signatures in the $B_{N}$ component relative to its background value were observed (panel 5f).

Returning to Fig. 4, the four bottom panels show an expansion of the Cluster ( $\mathrm{sc} 1$ and sc4) data, for the same period as TC-1 (12:10-12:40 UT). Again, panel (e) shows the PEACE/sc4 electron density, panels (f) and (g) show, respectively, the $B_{N}$ component and the magnitude of the FGM/sc4 magnetic field and panel (h) shows the 3 components of the CIS-HIA/sc1 ion velocity, in GSM coordinates. Once again, this expansion displays clearly the signatures of the injections: "reverse" $B_{N}$ bipolar signatures (panel $4 \mathrm{f}$ ) associated with decreases in the total magnetic field (panel $4 \mathrm{~g}$ ) and increases in the electron density (panels 4e). Finally, the ion velocity flow, which was mainly directed tailward, dawnward and southward in the HLBL, turned northward inside some of these injections, as evidenced by a slight reversal of the $V_{z}$ component (panel $4 \mathrm{~h}$ ). As for TC-1, we conclude that all these signatures are typical of FTE structures.

\section{c) Multi-spacecraft observations}

Inside the magnetosphere, all of the injections were observed by all four spacecraft and the order of entry (exit) into (from) each injection tube was the same throughout the period, i.e. 3-2-4-1. Therefore, the injection signatures observed by the four spacecraft were convective and not nested (see panel 5i). Due to the linear geometrical configuration of the spacecraft tetrahedron, it was not possible to determine precisely the direction of motion and the phase velocity of the injection tubes (Dunlop et al., 2002). However, knowing the timing and the order of entry into the injections tubes, we can infer one component of the direction of motion. As the $Z_{G S M}$ positions of all the spacecraft were very similar, a large uncertainty exists about the FTE motion (direction, velocity) in the $Z_{G S M}$ direction. On the other hand, we can deduce the direction of displacement in the $X Y_{G S M}$ plane, which was mainly tailward/duskward. The component of the velocity along the spacecraft line for the different FTEs varied between 50 and $100 \mathrm{~km} \mathrm{~s}^{-1}$.

\section{Discussion}

5.1 Interplanetary context of the TC-1 and Cluster observations and location of the reconnection site

In this subsection, we try to relate the evolution of plasma properties observed by Cluster and TC-1 over an extended period of $\sim 8.5 \mathrm{~h}$ near the dawnward magnetopause, with the changes in the interplanetary magnetic field orientation observed by Wind.

\section{a) Period 07:30-08:00 UT}

Between 07:30 and 08:00 UT, Cluster, located in the magnetosheath, observed four injections without high-energy magnetospheric plasma. Only magnetosheath electrons accelerated in the parallel direction were observed. A first explanation of these observations could be that these injections came from a reconnection site located on open field lines that were without magnetospheric plasma. In the parallel direction the magnetosheath plasma would then escape from the dawn southern lobe, after having been mirrored and after interaction with the reconnection site. However, before 08:20 UT, the IMF- $B_{z}$ component was 0 or slightly positive and the IMF- $B_{y}$ was negative (panels $\mathrm{b}$ and $\mathrm{c}$ of Fig. 2). For this IMF orientation, reconnection was more likely to take place at the duskside high-latitude magnetopause in the Southern Hemisphere. In order to observe reconnection signatures at Cluster, a long component reconnection line, extending from dusk to dawn along the southern lobe magnetopause, would be necessary. We have verified as to whether this reconnection geometry is possible by calculating the magnetic shear between the magnetosheath and magnetospheric field directions at the Cluster position. This shear is about $65^{\circ}$; such a reconnection geometry is possible, according to Phan et al. (1996). A second explanation would be that these injections came from a low-latitude reconnection site; the associated field lines would have been open for a long time, so that all the magnetospheric plasma would have already escaped. The IMF clock angle, which was highly variable before 08:20 UT, was above $70^{\circ}$ during these four reconnection signatures, consistent with a reconnection site located on the low-latitude magnetopause. After 08:20 UT, Cluster started to observe reconnected flux tubes containing magnetospheric plasma.

\section{b) Period 08:00-16:00 UT}

Despite a number of IMF variations, TC-1 observed reconnection signatures (FTEs) with similar characteristics throughout the entire period. Between 08:20 and 16:00 UT, the IMF- $B_{y}$ remained negative and relatively strong (except between 09:00 and 11:00 UT), favouring anti-parallel merging at a reconnection site located in the Northern Hemisphere, on the dawn flank of the magnetosphere. On the other hand, the IMF- $B_{z}$ was quite variable, oscillating between -4 and $+1 \mathrm{nT}$. In particular, between 08:20 and 11:00 UT the $B_{z}$ component was negative, and between 11:00 and 13:00 UT, the $B_{z}$ was slightly positive. Under these circumstances, the anti-parallel merging model would suggest that the reconnection site changed from a low-latitude site, where magnetosheath and closed magnetospheric field lines reconnect, to a high-latitude site, where magnetosheath and open lobe field lines may reconnect.

The observed FTEs signatures remained very similar between 08:00 and 16:00 UT for TC-1 and between 08:20 and 


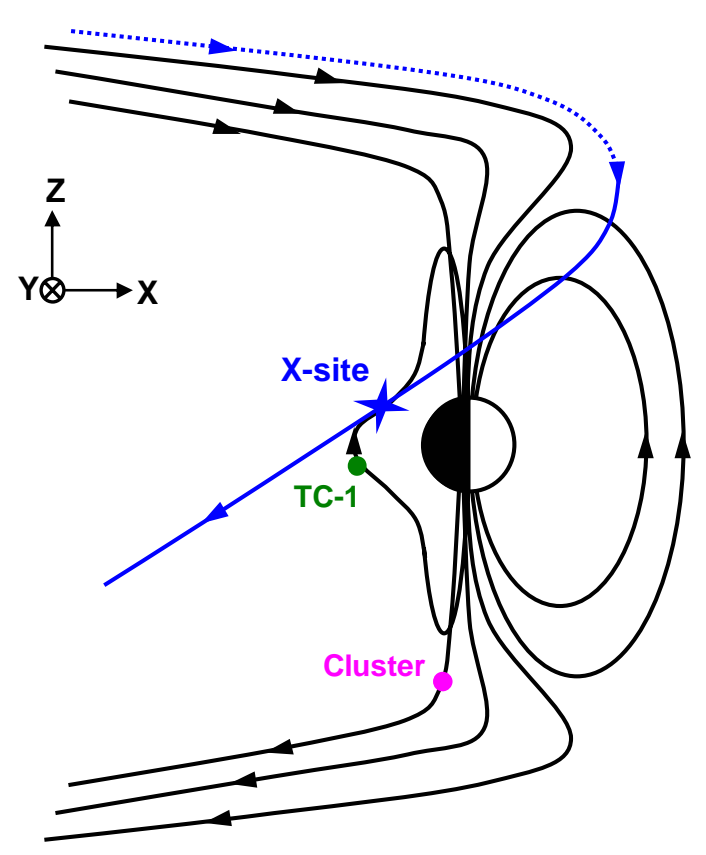

Fig. 6. Sketch in a meridian plane of the magnetosphere (Sun on the right of the figure). The reconnection site located at low latitude (X-site), as suggested in the Discussion, is represented by a blue cross. The approximate positions at $\sim 10: 00$ UT of Cluster- 1 and TC- 1 are also indicated by pink and green dots.

14:00 UT for Cluster. The detection of high-energy magnetospheric plasma mixed with low-energy magnetosheath plasma strongly suggests that reconnection was still occurring between the magnetosheath and closed magnetospheric field lines. The similar "reverse" FTE bipolar signatures observed by Cluster and TC- 1 imply that the reconnected flux tubes were moving southward from a common reconnection site (Rijnbeek and al., 1982). The flow acceleration inside the TC-1 flux tubes was in the same direction throughout the period 08:00-16:00 UT (tailward, southward and dawnward). Although the flow acceleration inside the injections observed by Cluster was not very clear, the order of entry of the four spacecraft inside each of the injections remained identical. These results suggested that the geometry of the reconnection was relatively stable throughout the period, with a single reconnection line probably located on the dawn flank of the magnetosphere and northward of both spacecraft, in agreement with expectations based on the negative IMF- $B_{y}$ orientation. A schematic of the magnetosphere seen from dawn with the position of the reconnection site is presented in Fig. 6. The small turnings of the IMF- $B_{z}$ component did not seem to significantly affect the location of the reconnection region, as might be expected on the basis of the anti-parallel merging model described above. If the reconnection site had changed from closed field lines to open lobe field lines, TC-1 should have stopped observing high-energy plasma of magnetospheric origin in the FTEs. Moreover, Cluster, located at $Z_{G S M} \sim-10 R_{E}$ in the Southern Hemisphere of the magneto- sphere (after 10:30 UT), would not be able to connect with northern lobe reconnected flux tubes and would stop detecting associated FTEs.

Freeman et al. (1993) and Senior et al. (2002) showed that dayside reconnection is not controlled simply by the IMF- $B_{z}$, but rather by the IMF clock angle. These authors concluded that dayside reconnection occurs when the clock angle is as low as $\sim 70^{\circ}$. Phan et al. (1996) and Sandholt et al. (1998) have even suggested a lower value of $\sim 45^{\circ}$ for this clock angle transition. Our results are in good agreement with these previous studies, since FTEs were always observed when the IMF clock angle was $\geq 70^{\circ}$, irrespective of the IMF- $B_{z}$ orientation. In summary, from our observations, it can be inferred that the reconnection site remained relatively stable in position and operated on closed field lines of the dawn flank of the magnetosphere between 08:00 and 16:00 UT (and maybe even during the 07:30-08:00 UT period), because the clock angle remained greater than $70^{\circ}$. This is a direct demonstration that the reconnection line can be stable in position for periods of at least 8 hours, despite variable IMF conditions.

\section{c) Disappearance of the FTEs signatures at TC-1 (10:55- 12:05 UT)}

Between 10:55 and 12:05 UT, the TC-1 reconnection signatures almost disappeared. However, some very faint signatures in the ion and electron spectrograms (panels b-e of Fig. 3), in the electron temperature and density (panels $\mathrm{f}$ and g of Fig. 3) were still observed. Moreover, the transition parameter $\tau$ (Hapgood and Bryant, 1990), applied to the TC-1 electron data, indicated that the spacecraft was located deeper inside the boundary layer during this period $(\tau>60)$, closer to the magnetosphere proper. During this period, the IMF- $B_{z}$ turned slightly positive and the solar wind pressure decreased slightly. We suggest two different possibilities (not necessarily incompatible) that could explain the disappearance of the reconnection signatures. First, a small inflation of the magnetosphere due to the decrease in the solar wind pressure moved TC-1 further inside the boundary layer of the magnetosphere, where the reconnection signatures may be harder to observe. Second, a decrease in the reconnection rate after the IMF- $B_{z}$ turned slightly positive implies a narrower reconnection boundary layer on the magnetopause, as well as a small inflation in the magnetosphere due to a decrease of magnetopause erosion. However, the complete interruption of reconnection is unlikely, as faint reconnection signatures were detected during this period and because reconnection signatures were still observed between 12:00 UT and $\sim$ 14:00 UT, despite IMF- $B_{z}$ orientation changes. Moreover, between at least 08:20 and 14:30 UT, Cluster also observed injections due to reconnection on closed field lines (mixing of high-energy magnetospheric plasma and lowenergy magnetosheath plasma) confirming the existence of a reconnection site at low latitude. 


\subsection{Sporadic nature of the reconnection}

As described in Sect. 4.1b, all the properties of the reconnection signatures observed by TC-1 were in agreement with previous observations of FTEs. We attribute all these events to a reconnection process acting in the dawn side of the magnetosphere, i.e. near the TC-1 location. Moreover, the increase in the total ion velocity observed inside the FTEs tubes (panel d of Fig. 4), higher than the magnetosheath flow velocity (by an amount of about the Alfvén velocity, $\mathrm{V}_{A}$ ), demonstrates that plasma acceleration was acting at the reconnection site.

At Cluster altitudes, all the properties of the injections (as described in Sect. 4.2b) were also in agreement with the observation of FTEs. Inside some of the injections, a reversal of the north-south component of the velocity was observed (from southward in the HLBL to northward inside the injections) (panel h of Fig. 4), in agreement with plasma precipitating along the field lines in the direction of the southern ionosphere. On the other hand, inside the magnetosphere (10:30 to 14:00 UT), each plasma injection was associated with a decrease in the total magnetic field, instead of an increase generally seen on the magnetopause. This result was probably due to the diamagnetic effect inside the magnetosphere (e.g. Bosqued et al., 2005). The convective signatures (panel i of Fig. 5) observed by the four spacecraft can only be explained by a drift motion of flux tubes inside the magnetosphere and not by incursions into the magnetosheath due to a back and forth motion of the magnetopause. Moreover, the direction of convection (tailward, southward and dawnward) deduced from the four-spacecraft magnetic field measurements was in agreement with flux tubes being dragged tailward along the magnetopause, under the action both of the magnetic tension forces at the reconnection site and the continuous magnetosheath flow.

As the Cluster spacecraft were aligned, it was not possible to determine a transverse size of the flux tubes from the multi-spacecraft measurements. However, by a single spacecraft method, we estimated this size by multiplying the ion velocity perpendicular to the magnetic field (convection velocity) by the time during which the spacecraft remained in the structure. We applied this method to 10 FTEs observed by TC- 1 and another 10 observed by Cluster/sc 4 . The perpendicular velocity inside the FTEs varied between 50 and $200 \mathrm{~km} \mathrm{~s}^{-1}$ and the length of time the spacecraft remained in the structure varied between 1 and $4 \mathrm{~min}$. The transverse sizes were therefore between 0.7 and $3.8 R_{E}$, indicating flux tubes of a finite and relatively small size, in agreement with previous work (Russell and Elphic, 1978; Saunders et al., 1984).

In summary, the Cluster and TC-1 observations strongly support the sporadic nature of the reconnection process, forming reconnected flux tubes of finite size (FTEs) and occurring throughout this long period (08:00-16:00 UT).
5.3 Comparison of observations between Cluster and Double Star

The FTEs observed by Cluster and TC- 1 were filled by a mixture of magnetospheric and magnetosheath plasma, even when observed by Cluster far inside the magnetosphere. Consequently, we suggest that these flux tubes were newlyreconnected and had probably not convected too far between the location of the reconnection site and the spacecraft positions. As Cluster and TC- 1 were separated by $\sim 2 \mathrm{~h}$ in MLT but observed simultaneously very newly-reconnected flux tubes, the reconnection line had to be extended by at least $2 \mathrm{~h}$ in MLT. On the other hand, no clear one-to-one correlation (even using a finite delay) was observed between the successive FTEs detected by Cluster and by TC-1, as seen in Fig. 4. This result could be due to the unknown convection time of the reconnected flux tubes between the reconnection site and Cluster, and between the reconnection site and TC-1, which introduces an ambiguity and makes the observations hard to compare. Some alternative explanations are described below. First, the reconnection rate may not be uniform along the length of the reconnection line. Cluster and TC- 1 could then observe flux tubes coming from different parts of the reconnection line. Second, TC-1, being close to the reconnection line, may observe all the freshly reconnected flux tubes, even if their directions of motion change due to the IMF variations. As Cluster is further from the reconnection line, the changes in direction in the flux tube motion would be more significant and Cluster could miss more of the FTE signatures. Finally, several reconnection lines could co-exist on the dawnside flank (Berchem et al., 1995). Cluster and TC-1 could then observe FTEs coming from different reconnection lines. The fact that the recurrence period was shorter at TC-1 than at Cluster (4 min against $8 \mathrm{~min}$ ) could support these different ideas. All of these points will be addressed in a future study.

\section{Conclusions}

On 8 May 2004, between 08:00 and 16:00 UT, the TC-1 and Cluster spacecraft were located at low- and high-latitudes, respectively, on the southern dawn flank of the magnetosphere. Both spacecraft observed clear and successive FTE signatures, with "reverse" bipolar signatures of the magnetic field normal component and with a repetition rate of 4 (TC-1) to 8 (Cluster) minutes. These conjugate observations suggest that reconnection was occurring at a reconnection site located northward of both TC-1 and Cluster. Despite variable IMF conditions (particularly changes in the IMF- $B_{z}$ orientation), the reconnection site was relatively stable in location throughout the period and remained on closed magnetospheric field lines, presumably as the IMF clock angle was always $\geq 70^{\circ}$.

The very clear signatures and the finite transverse sizes of the FTEs, observed both by TC- 1 and Cluster, suggest the sporadic nature of the reconnection process, even for a period 
as long as $\sim 8 \mathrm{~h}$. The simultaneity of the FTE observations by TC- 1 and Cluster implies that the reconnection line was extended over at least $2 \mathrm{~h}$ in MLT. However, no one-to-one correlation was observed between the FTEs seen by Cluster and by TC-1.

Acknowledgements. This work was sponsored by the UK Particle Physics and Astronomy Research Council. The authors thank the Cluster and Double Star PEACE, FGM and CIS/HIA operations team and acknowledge the principal investigator R. Lepping and CDAWeb for providing the Wind magnetic field data.

Topical Editor T. Pulkkinen thanks S. Milan and another referee for their help in evaluating this paper.

\section{References}

Balogh, A., Carr, C. M., Acuña, M. H., et al.: The Cluster magnetic field investigation: overview of in-flight performance and initial results, Ann. Geophys., 19, 1207-1217, 2001,

\section{SRef-ID: 1432-0576/ag/2001-19-1207.}

Berchem, J., Raeder, J., and Ashour-Abdalla, M.: Magnetic flux ropes at the high-latitude magnetopause, Geophys. Res. Lett., 22, 1189-1192, 1995.

Berchem, J. and Russell, C. T.: Flux transfer events on the magnetopause: spatial distribution and controlling factors, J. Geophys. Res., 89, 6689-6703, 1984.

Bosqued, J. M., Phan, T. D., Dandouras, I., et al.: CLUSTER observations of the high-latitude magnetopause and cusp: initial results from the CIS ion instruments, Ann. Geophys., 19, 15451566, 2001,

\section{SRef-ID: 1432-0576/ag/2001-19-1545.}

Bosqued, J. M., Escoubet, C. P., Frey, H. U., Dunlop, M. W., Berchem, J., Marchaudon, A., Cerisier, J.-C., Fazakerley, A. N., Budnik, E., Lavraud, B., Rème, H., Laakso, H., and Balogh, A.: Multipoint observations of transient reconnection signatures in the cusp precipitation: A CLUSTERIMAGE detailed case study, J. Geophys. Res., 110, A03219, doi:10.1029/2004JA010621, 2005.

Chandler, M. O., Fuselier, S. A., Lockwood, M., and Moore, T. E.: Evidence of component merging equatorward of the cusp, J. Geophys. Res., 104, 22 623-22 633, 1999.

Crooker, N. U.: Dayside merging and cusp geometry, J. Geophys. Res., 84, 951-959, 1979.

Dungey, J. W.: Interplanetary magnetic field and the auroral zones, Phys. Rev. Lett., 6, 47-48, 1961.

Dungey, J. W.: The Structure of the Exosphere or Adventures in Velocity Space, lecture delivered at Les Houches during the 1962 session of the Summer Scholl of Theoretical Physics University of Grenoble, in: The Earth's Environment, edited by: DeWitt, C., Hieblot, J., and Lebeau, A., Gordon and Breach, New York, 1963.

Dunlop, M. W., Balogh, A., and Glassmeier, K.-H: Fourpoint Cluster application of magnetic field analysis tools: the discontinuity analyzer, J. Geophys. Res., 107 (A11), 1385, doi:10.1029/2001JA005089, 2002.

Farrugia, C. J., Rijnbeek, R. P., Saunders, M. A., Southwood, D. J., Rodgers, D. J., Smith, M. F., Chaloner, C. P., Hall, D. S., Christiansen, P. J., and Woolliscroft, L. J. C.: A multi-instrument study of flux transfer event structure, J. Geophys. Res., 93, 1446514477, 1988.
Fazakerley, A. N., Carter, P. J., Watson, G., Spencer, A., Sun, Y. Q., Kataria, D. O., Fontaine, D., Liu, Z. X., Gilbert, L., He, L., Lahiff, A. D., Mihaljcic, B., Szita, S., Taylor, M. G. G. T., Wilson R. J., and Schwartz, S. J.: The Double Star Plasma Electron and Current Experiment, Ann. Geophys., 23, 2733-2756, 2005.

Freeman, M. P., Farrugia, C. J., Burlaga, L. F., Hairston, M. R., Greenspan, M. E., Ruohoniemi, J. M., and Lepping, R. P.: The interaction of a magnetic cloud with the Earth: Ionospheric convection in the northern and southern hemispheres for a wide range of quasi-steady interplanetary magnetic field conditions, J. Geophys. Res., 98, 7633-7655, 1993.

Fuselier, S. A., Trattner, K. J., and Petrinec, S. M.: Cusp observations of high- and low-latitude reconnection for northward interplanetary magnetic field, J. Geophys. Res., 105, 253-266, 2000.

Gosling, J. T., Asbridge, J. R., Bame, S. J., Feldman, W. C., Paschmann, G., Sckopke, N., and Russell, C. T.: Evidence for quasi-stationary reconnection at the dayside magnetopause, J. Geophys. Res., 87, 2147-2158, 1982.

Gosling, J. T., Thomsen, M. F., Bame, S. J., Elphic, R. C., and Russell, C. T.: Observations of reconnection of interplanetary and lobe magnetic field lines at the high-latitude magnetopause, J. Geophys. Res., 96, 14 097-14 106, 1991.

Hapgood, M. A., and Bryant, D. A.: Re-ordered electron data in the low-latitude boundary layer, Geophys. Res. Lett., 17, 20432046, 1990.

Johnstone, A. D., Alsop, C., Burge, S., Carter, P. J., Coates, A. J., Coker, A. J., Fazakerley, A. N., Grande, M., Gowen, R. A., Gurgiolo, C., Hancock, B. K., Narheim, B., Preece, A., Sheather, P. H., Winningham, J. D., and Woodliffe, R. D.: Peace: A Plasma Electron and Current Experiment, Space Sci. Rev. 79, 351-398, 1997.

Lee, L. C. and Fu, Z. F.: A theory of magnetic flux transfer at the Earth's magnetopause, Geophys. Res. Lett., 12, 105-108, 1985.

Lockwood, M., Cowley, S. W. H., Sandholt, P. E., and Lepping, R. P.: The ionospheric signatures of flux transfer events and solar wind dynamic pressure changes, J. Geophys. Res., 95, $17113-$ $17135,1990$.

Lockwood, M. and Wild, M. N.: On the quasi-periodic nature of magnetopause flux transfer, J. Geophys. Res., 98, 5935-5940 1993.

Maezawa, K.: Magnetospheric convection induced by the positive and negative $\mathrm{Z}$ components of the interplanetary magnetic field: quantitative analysis using polar cap magnetic records, J. Geophys. Res., 81, 2289-2303, 1976.

Marcucci, M. F., Bavassano Cattaneo, M. B., Di Lellis, A. M., Irelli, P. C., Kistler, L. M., Phan, T. D., Haerendel, G., Klecker, B., Paschmann, G., Baumjohann, W., Mobius, E., Popecki, M. A., Sauvaud, J. A., Rème, H., Korth, A., Eliasson, L., Carlson, C. W., McCarthy, M., and Parks, G. K.: Evidence for interplanetary magnetic field B-y controlled large-scale reconnection at the dayside magnetopause, J. Geophys. Res., 105, 27 497-27 507, 2000.

Milan, S. E., Lester, M., Cowley, S. W. H., and Brittnacher, M.: Convection and auroral response to a southward turning of the IMF: Polar UVI, CUTLASS, and IMAGE signatures of transient magnetic flux transfer at the magnetopause, J. Geophys. Res., 105, 15 741-15 755, 2000.

Owen, C. J., Fazakerley, A. N., Carter, P. J., Coates, A. J., Krauklis, I. C., Szita, S., Taylor, M. G. G. T., Travnicek, P., Watson, G., Wilson, R. J., Balogh, A., and Dunlop, M. W.: CLUSTER PEACE observations of electrons during magnetosheric flux transfer events, Ann. Geophys., 19, 1509-1522, 2001, 
SRef-ID: 1432-0576/ag/2001-19-1509.

Paschmann, G., Haerendel, G., Papamastorakis, I., Sckopke, N., Bame, S. J., Gosling, J. T., and Russell, C. T.: Plasma and magnetic field characteristics of magnetic flux transfer events, J. Geophys. Res., 87, 2159-2168, 1982.

Phan, T. D., Dunlop, M. W., Paschmann, G., Klecker, B., Bosqued, J.-M., Rème, H., Balogh, A., Twitty, C., Mozer, F. S., Carlson, C. W., Mouikis, C., and Kistler, L. M.: Cluster observations of continuous reconnection at the magnetopause under steady interplanetary magnetic field conditions, Ann. Geophys., 22, 23552367, 2004,

SRef-ID: 1432-0576/ag/2004-22-2355

Phan, T. D., Kistler, L. M., Klecker, B., et al.: Extended magnetic reconnection at the Earth's magnetopause from detection of bidirectional jets, Nature, 404, 848-850, 2000.

Phan, T. D., Paschmann, G., and Sonnerup, B. U. Ö.: Low-latitude dayside magnetopause and boundary layer for high magnetic shear 2. Occurrence of magnetic reconnection, J. Geophys. Res., 101, 7817-7828, 1996.

Phan, T. D., Sonnerup, B. U. O., and Lin, R. P.: Fluid and kinetic signatures of reconnection at the dawn tail magnetopause: Wind observations, J. Geophys. Res., 106, 25 489-25 502, 2001.

Pinnock, M., Chisham, G., Coleman, I. J., Freeman, M. P., Hairston, M., and Villain, J.-P.: The location and rate of dayside reconnection during an interval of southward interplanetary magnetic field, Ann. Geophys., 21, 1467-1482, 2003,

SRef-ID: 1432-0576/ag/2003-21-1467.

Pinnock, M., Rodger, A. S., Dudeney, J. R., Rich, F., and Baker, K. B.: High spatial and temporal resolution observations of the ionospheric cusp, Ann. Geophys., 13, 919-925, 1995,

SRef-ID: 1432-0576/ag/1995-13-919.

Pitout, F., Newell, P. T., and Buchert, S. C.: Simultaneous high- and low-latitude reconnection: ESR and DMSP observations, Ann. Geophys., 20, 1311-1320, 2002,

SRef-ID: 1432-0576/ag/2002-20-1311

Rème, H., Aoustin, C., Bosqued, J.-M., et al.: First multispacecraft ion measurements in and near the earth's magnetosphere with the identical CLUSTER Ion Spectrometry (CIS) Experiment, Ann. Geophys., 19, 1303-1354, 2001,

SRef-ID: 1432-0576/ag/2001-19-1303
Rijnbeek, R. P., Cowley, S. W. H., Southwood, D. J., and Russell, C. T.: Observations of reverse polarity flux transfer events at the earth's dayside magnetopause, Nature, 300, 23-26, 1982.

Roelof, E. C. and Sibeck, D. G.: Magnetopause shape as a bivariate function of interplanetary magnetic field and solar wind dynamic pressure, J. Geophys. Res., 98, 21 421-21 450, 1993.

Russell, C. T. and Elphic, R. C.: Initial ISEE magnetometer results: magnetopause observations, Space Sci. Rev., 22, 681-715, 1978.

Russell, C. T., Le, G., Kawano, H., Petrinec, S. M., and Zhang, T. L.: Intrinsic time scale for reconnection on the dayside magnetopause, Adv. Space Res., 19, 1913-1917, 1997.

Sandholt, P. E., Farrugia, C. J., Moen, J., Noraberg, Ø., Lybekk, B., Sten, T., and Hansen, T.: A classification of dayside auroral forms and activities as a function of interplanetary magnetic field orientation, J. Geophys. Res., 103, 23 325-23 345, 1998.

Saunders, M. A., Russell, C. T., and Sckopke, N.: Flux transfer events: scale size and interior structure, Geophys. Res. Lett., 11, 131-134, 1984.

Senior, C., Cerisier, J.-C., Rich, F. J., Lester, M., and Parks, G. K.: Strong sunward propagating flow bursts in the night sector during quiet solar wind conditions: SuperDARN and satellite observations, Ann. Geophys., 20, 771-779, 2002,

SRef-ID: 1432-0576/ag/2002-20-771.

Shue, J.-H., Chao, J. K., Fu, H. C., Russell, C. T., Song, P., Khurana, K. K, and Singer, H. J.: A new functional form to study the solar wind control of the magnetopause size and shape, J. Geophys. Res., 102, 9497-9511, 1997.

Sonnerup, B. U.: Magnetopause Reconnection Rate, J. Geophys. Res., 79, 1546-1549, 1974.

Vaisberg, O. L., Avanov, L. A., Moore, T. E., and Smirnov, V. N.: Ion velocity distributions within the LLBL and their possible implication to multiple reconnections, Ann. Geophys., 22 213-236, 2004,

SRef-ID: 1432-0576/ag/2004-22-213.

Wild, J. A., Milan, S. E., Cowley, S. W. H., Bosqued, J. M., Rème, H., Nagai, T., Kokubun, S., Saito, Y., Mukai, T., Davies, J. A., Cooling, B. M. A., Balogh, A., and Daly, P. W.: Simultaneous insitu observations of the signatures of dayside reconnection at the high- and low-latitude magnetopause, Ann. Geophys., 23, 445460, 2005,

SRef-ID: 1432-0576/ag/2005-23-445 